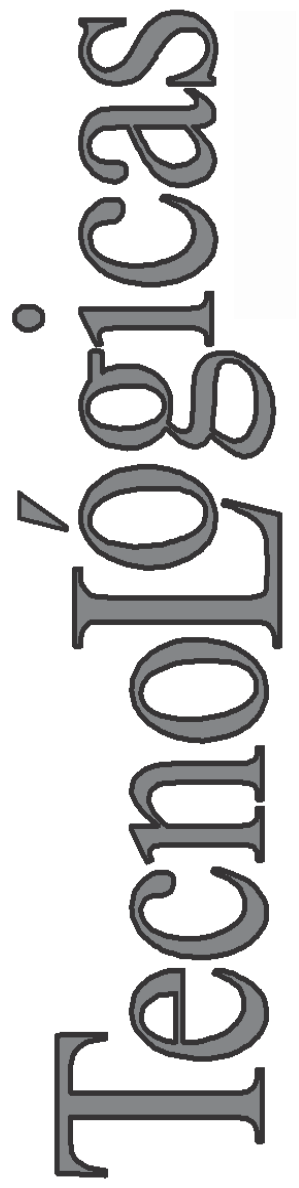

\title{
Método de Calibración para Medir el Índice de Reproducción Cromática (CRI) usando Sensores RGB
}

\section{Calibration Method for Measuring the Color Rendering Index (CRI) using RGB Sensor}

\author{
Juan S. Botero-Valencia ${ }^{1}$ \\ Francisco E. López-Giraldo ${ }^{2}$ \\ Jesús F. Vargas-Bonilla ${ }^{3}$
}

1 Grupo Automática, Electrónica y Ciencias Computacionales, Facultad de Ingenierías, Instituto

Tecnológico Metropolitano, Medellín-Colombia juanbotero@itm.edu.co

2 Grupo Automática, Electrónica y Ciencias Computacionales, Facultad de Ingenierías, Instituto

Tecnológico Metropolitano, Medellín-Colombia franciscolopez@itm.edu.co

3 Grupo Sistemic, Facultad de Ingeniería, Universidad de Antioquia, Medellín-Colombia jfvargas@udea.edu.co 


\title{
Resumen
}

El color es una característica perceptiva derivada de la luz que se refleja sobre los objetos y como tal no representa una propiedad física. Sin embargo, se han realizado avances en la construcción de modelos matemáticos que permitan definirlo con mayor claridad. Las fuentes de luz juegan un papel fundamental en la percepción de los colores y una de las medidas para caracterizar dicho efecto es el Índice de Reproducción Cromática (CRI). El CRI es una medida usada para caracterizar la capacidad de reproducción del color de una fuente de luz en particular en comparación con una fuente de luz ideal. En este trabajo, inicialmente se desarrolló un algoritmo para determinar el CRI a partir del SPD (Distribución Espectral de Potencia) y posteriormente se utilizó un sensor RGB de bajo costo para encontrar un modelo que permitiera estimar el valor CRI directamente. Se usaron cuatro grupos de fuentes de luz con información técnica disponible, se midió el SPD y se encontró una matriz de transformación para convertir el espacio RGB al XYZ. Este trabajo está enmarcado en el desarrollo de herramientas que permitan controlar y realizar medidas de iluminación con el propósito de realizar tareas de control de ambientes y caracterización de fuentes artificiales.

\section{Palabras clave}

Control de ambientes; calibración; índice de rendimiento de color; iluminación; sensor de color RGB.

\begin{abstract}
Color is a perceptual characteristic, derived from the light reflected on the object and as such does not represent a physical property. However, progress has been made in building mathematical models to define it more clearly. Light sources play a key role in color perception, and one of the measures to characterize the effect is the Color Rendering Index (CRI). The CRI is a measure used to characterize the color reproduction ability of a light source in particular in comparison with ideal light source. In this work, initially developed an algorithm to determine the CRI from SPD and then used a lowcost RGB sensor to find a model that would estimate the value CRI directly. We used four groups of light sources with technical information available, we measured the SPD and found a transformation matrix to convert RGB to XYZ space. This work is framed in the development of tools to control and lighting measurements for environment control tasks and characterization of artificial sources.
\end{abstract}

\section{Keywords}

Ambient controt; calibration; color rendering index; illumination; RGB color sensor. 


\section{INTRODUCCIÓN}

La luz representa una parte fundamental en la interpretación del color, se refleja sobre los objetos y es absorbida por los receptores en la retina para formar dentro del cerebro la percepción que tenemos del color. Se han realizado avances para construir modelos matemáticos que permitan describir el color y las fuentes de luz, entre los más importantes se encuentran los realizados por la International Commission on Illumination (CIE por sus siglas en francés). Una de las medidas para caracterizar las fuentes es el Índice de Rendimiento de Color (CRI). El CRI es una medida usada para representar la reproducibilidad de colores de una fuente de luz. Se deriva del cálculo del CCT que representa la temperatura que tendría (en grados Kelvin) un cuerpo negro con la misma emisión lumínica (Sharma, 2003).

El color como propiedad es utilizado en numerosas aplicaciones, desde determinar la elaboración de zumos de naranja (Fernández-Vázquez et al., 2010), para verificar la estabilidad en salchichas (Amensour et al., 2010), para segmentar imágenes en secuencias de video (Botero Valencia \& Delgado Trejos, 2009) y para clasificar calidad en frutas como en (Motonaga et al., 2010) entre muchas otras aplicaciones. En general la percepción del color es una propiedad que aportó ventajas evolutivas a nuestra especie (Lopera, 2004). La importancia de las fuentes de luz es innegable en la caracterización del color y como tal se han se han realizado trabajos académicos de gran utilidad donde se caracterizan fuentes de luz como en (Elvidge et al., 2010) donde se utilizó información del espectro para obtener características derivadas desde las fuentes de combustible líquido hasta los LED. De igual forma se han realizado trabajos de caracterización específica de fuentes LED (Lei et al., 2007).

El índice CRI es una medida (Bouma, 1948) usada para representar la reproducibilidad perceptiva de colores de una fuente, podría verse como la cercanía al blanco de una fuente de luz en particular. El uso de este índice aún se encuentra en discusión y se han realizado trabajos para compararlo con índices más complejos (Rea \& Freyssinier-Nova, 2008). Sin embargo, este parámetro es usado para determinar diferentes tipos de atmósferas comerciales 
(ASSIST, 2010), las fuentes con valores CRI altos reproducen con mejor calidad los colores y mejoran la percepción de los productos. Actualmente un valor aproximado del CRI se encuentra disponible en la información técnica que entregan los fabricantes de luminarias. Adicionalmente se han realizado trabajos para determinar la respuesta de usuarios a cambios realizados en ambientes controlados donde se varia el CCT y el CRI (Logadóttir et al., 2013) y se evalúa su nivel de aceptación.

La mejor forma de estimar el índice CRI de una fuente es usar la información espectral (SPD) para determinar los valores en el espacio XYZ y usar los valores presentados en (McCamy, 1992) y calcular el valor CCT. Con el valor CCT derivar el espectro de referencia de la fuente para posteriormente derivar parámetros tanto para el SPD de referencia y el de la fuente usando las curvas TCS definidas por el CIE, finalmente se calcula la distancia euclidea y se estima el valor CRI. El uso de espectrometría complica el modelo de implementación y dificulta su utilización dinámica en aplicaciones comunes de control de iluminación (Pandharipande \& Caicedo, 2011), (Ashibe et al., 2008), (Sandhu et al., 2004), es indiscutible que puede sería útil adicionar modelos de control CRI a dichos sistemas.

En este trabajo se presenta una metodología de calibración que permite realizar una estimación de CRI usando un sensor RGB de bajo costo. Para esto, se caracterizaron cuatro grupos de fuentes comunes que permitieron estimar una matriz para transformar el espacio RGB de respuesta del sensor a un espacio XYZ normalizado, con dicha información se pudo encontrar un.modelo de mínimos cuadrados. La aproximación realizada arroja un error inferior al 6\%. Esta iniciativa está enmarcada en labores de investigación que buscan construir modelos simples que permitan mejorar de forma automática las características de iluminación y por consiguiente de percepción del color en ambientes comunes y en aplicaciones de control de calidad en procesos industriales. 


\section{MATERIALES Y MÉTODOS}

\subsection{Espectrómetro LR1}

El LR1 Es un espectrómetro de bajo costo desarrollado por ASEQ Instruments con un sensor CCD (Charge Coupled Device) lineal TOSHIBA. Las características más relevantes se presentan en la Tabla 1.

Tabla 1. Características LR1

\begin{tabular}{llc}
\hline \multicolumn{1}{c}{ Característica } & \multicolumn{1}{c}{ Valor } & Unidades \\
\hline Sensor & Toshiba TCD1304AP & {$[\mathrm{NA}]$} \\
Rango del detector & $200-1100$ & {$[\mathrm{~nm}]$} \\
Pixeles & 3648 & {$[\mathrm{px}]$} \\
Tamaño del Pixel & $8 \times 200$ & {$[\mu \mathrm{m}]$} \\
Profundidad de pixel & 100000 & {$[$ electrons $]$} \\
Resolución A/D & 14 & {$[\mathrm{bit}]$} \\
Tiempo de exposición & $2.5-10000$ & {$[\mathrm{~ms}]$} \\
Tiempo de lectura CCD & 14 & {$[\mathrm{~ms}]$} \\
Comunicación & USB 2.0 & {$[\mathrm{NA}]$} \\
\hline
\end{tabular}

\subsection{TCS3414CS}

El TCS3414CS es un sensor de color fabricado por Texas, Advanced Optoelectrónicos Solutions (TAOS). Está construido con un arreglo de 16 fototodiodos filtrados (8x2), cuatro de los cuales tienen filtros rojos, cuatro azules y cuatro verdes, los restantes cuatro no se filtran. Cada uno de los cuatro canales del sensor (rojo, verde, azul, sin filtrar) entrega su salida en formato de 16 bits en protocolo I2C a $400 \mathrm{KHz}$. La ganancia del convertidor analógico y el tiempo de integración son programables. El sensor tiene una entrada de sincronización (SYNC), que permite controlar con precisión la integración de fuentes externas. La Tabla 2 muestra algunas características importantes del TCS3414CS. Un filtro interno elimina la fluctuación de la señal debido al parpadeo AC. 
Tabla 2. Características TCS3414CS

\begin{tabular}{llc}
\hline \multicolumn{1}{c}{ Característica } & \multicolumn{1}{c}{ Valor } & Unidades \\
\hline Sensor & Fotodiodo & {$[\mathrm{NA}]$} \\
Frecuencia de reloj & $0-400$ & {$[\mathrm{KHz}]$} \\
Resolución A/D & 16 & {$[\mathrm{bits}]$} \\
Voltaje de operación & $2.7-3.6$ & {$[\mathrm{~V}]$} \\
Corriente $\left(\mathrm{V}_{\mathrm{DD}}=3.6\right)$ & $8.7-11$ & {$[\mathrm{~mA}]$} \\
Comunicación & $\mathrm{I} 2 \mathrm{C}$ & {$[\mathrm{NA}]$} \\
Canales & $\mathrm{R}, \mathrm{G}, \mathrm{B}$, clear & {$[\mathrm{NA}]$} \\
\hline
\end{tabular}

\subsection{CIE Standard Observer Matching Functions}

Las curvas CIE $\tilde{x}(\lambda), \tilde{y}(\lambda)$, y $\tilde{z}(\lambda)$ son funciones que representan numéricamente la respuesta de un observador estándar, se muestran en la Fig. 1. Estas funciones pueden ser vistas como la sensibilidad de tres detectores lineales que produce el valor CIE triestímulo $X, Y$ y $Z$. El conjunto de funciones son conocidas como el estándar del observador. En (CIE, 2013) se definen las "CIE standard colorimetric observer tables" que son usadas para los cálculos en este artículo.

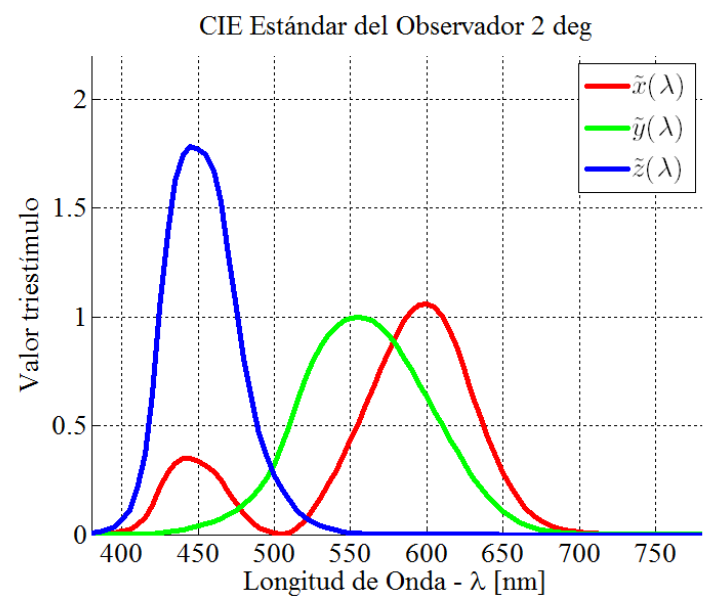

Fig. 1. CIE Estándar del observador 2 deg 


\subsection{Cálculo del CRI}

Las coordenadas CIE $X, Y$ y $Z$.de una fuente de luz pueden ser calculadas usando la Distribución Espectral de Potencia (SPD) de la fuente y usando las "CIE standard observer matching functions". Las ecuaciones son presentadas en la ecuación (1). En (1), $K$ es una constante para normalizar $Y$ a 100.

$X=K \sum_{\lambda=380}^{780} \operatorname{SPD}(\lambda) \cdot \tilde{x}(\lambda), Y=K \sum_{\lambda=380}^{780} \operatorname{SPD}(\lambda) \cdot \tilde{y}(\lambda), Z=K \sum_{\lambda=380}^{780} \operatorname{SPD}(\lambda) \cdot \tilde{z}(\lambda)$

Con los valores $X, Y$ y $Z$ se pueden obtener las coordenadas de cromaticidad $x, y$. Las ecuaciones se presentan en (2).

$x=\frac{X}{X+Y+Z}, \quad y=\frac{Y}{X+Y+Z}$

Posteriormente con las ecuaciones presentadas en (McCamy, 1992), se estima la Temperatura Correlacionada de Color (CCT). Las ecuaciones se presentan en (3).

$C C T=449 n^{3}+3525 n^{2}+6823 n+5520, \quad n=\frac{x-0.3320}{0.1858-y}$

El paso siguiente es determinar la iluminación de referencia con base en el CCT de la fuente de luz. La iluminación de referencia tiene el mismo CCT que la fuente de iluminación de interés. Se calcula usando la ecuación (4) si el valor de CCT $<5000 \mathrm{~K}$ o el modelo presentado en (5) en caso contrario.

$S P D_{\text {Ref }}=2 \pi h c^{2}\left(10^{-9} \lambda\right)^{-5} /\left(e^{\frac{\left[\left(\frac{h c}{k}\right)\right]}{\left[\left(C C T 10^{-9} \lambda\right)\right]}}-1\right)$

$h=6.626069 \times 10^{-34} \mathrm{~J} . \mathrm{s}$

$c=2.997924 \times 10^{8} \mathrm{~m} / \mathrm{s}$

$k=1.3806504 \times 10^{-23} \mathrm{~J} / K$

En caso de que el $C C T \geq 5000 \mathrm{~K}$, la iluminación de referencia puede ser calculada como se muestra en (5). 
$S P D_{\text {Ref }}(\lambda)=S_{0}(\lambda)+\left[M_{1} \times S_{1}(\lambda)\right]+\left[M_{2} \times S_{2}(\lambda)\right]$

$S_{0}(\lambda), S_{1}(\lambda)$ y $S_{2}(\lambda)$ son vectores de distribución de luz del día

$M_{1}=\frac{-1.3515-1.7703 x_{D}+5.9114 y_{D}}{0.0241+0.2562 x_{D}-0.7341 y_{D}}$

$M_{2}=\frac{0.0300-31.4424 x_{D}+30.0717 y_{D}}{0.0241+0.2562 x_{D}-0.7341 y_{D}}$

$x_{D}=\frac{-4.6070 \times 10^{9}}{T_{C}^{3}}+\frac{2.9678 \times 10^{6}}{T_{C}^{2}}+\frac{0.09911 \times 10^{3}}{T^{3}}+0.244063$

si $\mathrm{CCT} \leq 7000 \mathrm{~K}$

$x_{D}=\frac{-2.0064 \times 10^{9}}{T_{C}^{3}}+\frac{1.9018 \times 10^{6}}{T_{C}^{2}}+\frac{0.24748 \times 10^{3}}{T^{3}}+0.237040$

si $7000 \mathrm{~K}<\mathrm{CCT} \leq 25000 \mathrm{~K}$

$y_{D}=-3.000\left(x_{D}\right)^{2}+2.870 x_{D}-0.275$

Se determina los valores del CIE $1960(\mathrm{u}, \mathrm{v})$ para cada uno de las ocho muestras de color (TCS) tanto la fuente de interés como la iluminación de referencia y a para cada uno los valores $(X, Y$ y $Z$ ) como se muestra en (6).

$$
\begin{aligned}
& X_{i}=K \sum_{\lambda=380}^{780} S P D(\lambda) \cdot \tilde{x}(\lambda) \cdot T C S_{i}(\lambda) d \lambda \\
& Y_{i}=K \sum_{\lambda=380}^{780} \operatorname{SPD}(\lambda) \cdot \tilde{y}(\lambda) \cdot T C S_{i}(\lambda) d \lambda \\
& Z_{i}=K \sum_{\lambda=380}^{780} \operatorname{SPD}(\lambda) \cdot \tilde{z}(\lambda) \cdot \operatorname{TCS}_{i}(\lambda) d \lambda
\end{aligned}
$$

Se deriva los valores CIE $1960(\mathrm{u}, \mathrm{v})$ para cada TCS usando (7).

$u=\frac{4 X}{X+15 Y+3 Z}, \quad v=\frac{6 X}{X+15 Y+3 Z}$

Se aplica el corrimiento de color adaptativo de von Kries para tener en cuenta las diferencias de estados de adaptación cromática 
entre la fuente de luz de interés y la iluminación de referencia como se muestra en (8).

$$
\begin{aligned}
& c=\frac{1}{v}(4-u-10 v), \quad d=\frac{1}{v}(1.708 v+0.404-1.481 u) \\
& u_{k, i}=\frac{10.872+0.404 \frac{c_{r e f}}{c_{k}} c_{k, i}-4 \frac{d_{r e f}}{d_{k, i}} d_{k, i}}{16.518+1.481 \frac{c_{r e f}}{c_{k}} c_{k, i}-\frac{d_{r e f}}{d_{k, i}} d_{k, i}} \\
& v_{k, i}=\frac{5.520}{16.518+1.481 \frac{c_{r e f}}{c_{k}} c_{k, i}-\frac{d_{r e f}}{d_{k, i}} d_{k, i}}
\end{aligned}
$$

Se determina los valores CIE $1964 \mathrm{~W} * \mathrm{U} * \mathrm{Vi}$ para cada TCS (9).

$$
\begin{gathered}
W_{i}^{*}=25\left(Y_{i}\right)^{1 / 3}-17, \quad U_{i}^{*}=13\left(W_{i}\right)\left(u_{i}-u\right), \\
V_{i}^{*}=13\left(W_{i}^{*}\right)\left(v_{i}-v\right)
\end{gathered}
$$

Por último se determina el corrimiento de color $\Delta E$ para cada TCS con la ecuación (10).

$$
\Delta E_{i}=\sqrt{\left(U_{r e f, i}^{*}-U_{k, i}^{*}\right)^{2}+\left(V_{r e f, i}^{*}-V_{k, i}^{*}\right)^{2}+\left(W_{r e f, i}^{*}-W_{k, i}^{*}\right)^{2}}
$$

Se determina el CRI individual con (11).

$R_{i}=100-4.6 \Delta E$

Y finalmente el CRI general (12).

$$
R_{a}=\frac{1}{8} \sum_{1}^{8} R_{i}
$$

\section{RESULTADOS Y DISCUSIONES}

Inicialmente para calcular los valores CRI de las fuentes, se obtuvieron con el espectrómetro LR1 las SPD. En la Fig. 2a se 
muestran las SPD de las fuentes LED y en la Fig. $2 \mathrm{~b}$ de las fuentes fluorescentes.

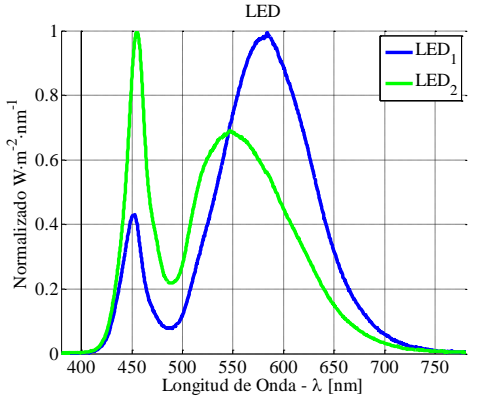

a)

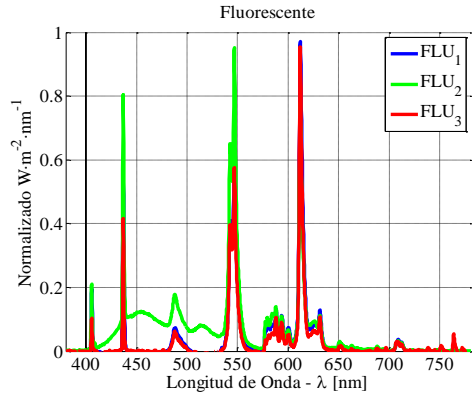

b)

Fig. 2. SPD de las fuentes a) LED y b) Fluorescentes

En la Fig. 3a se presenta la SPD de luminarias incandescentes con filamento de wolframio y en la Fig. $3 \mathrm{~b}$ de fuentes incandescentes halógenas.

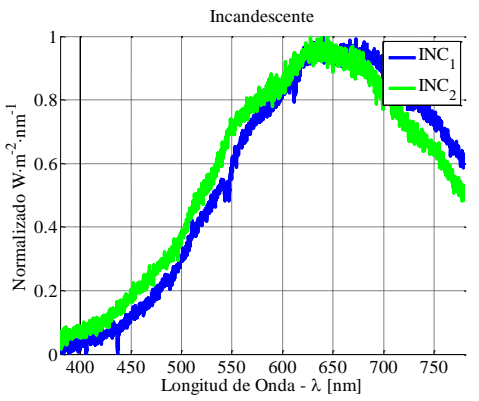

a)

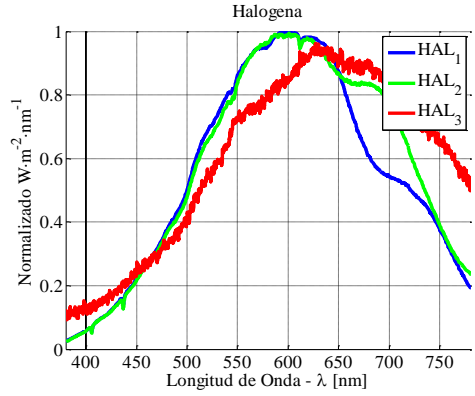

b)

Fig. 3. SPD de las fuentes a) incandescentes y b) halógenas

Con la información obtenida se pueden estimar los parámetros de la matriz $T$ que se presenta en (13). El valor $X, Y, Z$ es el valor de las coordenadas en el espacio CIE y el vector $R, G, B$ los valores de salida del sensor.

$\left[\begin{array}{l}X \\ Y \\ Z\end{array}\right]=T\left[\begin{array}{l}R \\ G \\ B\end{array}\right]=\left[\begin{array}{lll}a_{1,1} & a_{1,2} & a_{1,3} \\ a_{2,1} & a_{2,2} & a_{2,3} \\ a_{3,1} & a_{3,2} & a_{3,3}\end{array}\right]\left[\begin{array}{l}R \\ G \\ B\end{array}\right]$ 
Para calcular $T$ se usa (14), donde $\left[X_{R} Y_{R} Z_{R}\right]_{3 \times 10}$ representa una matriz construida con los valores reales CIE $X, Y$ y $Z$ y $[R G B]_{3 \times 10}$ es una matriz de igual tamaño construida con los valores medidos con el sensor $R, G, B$. La ecuación para encontrar $T$ y la matriz resultante se presenta en (14).

$\begin{aligned} T & =\left[X_{R} Y_{R} Z_{R}\right]_{3 \times 10} \operatorname{pinv}\left([R G B]_{3 \times 10}\right) \\ T & =\left[\begin{array}{ccc}0.3570 & 0.0263 & 0.1029 \\ 0.2809 & 0.0967 & 0.1520 \\ -0.0763 & 0.0120 & 0.5673\end{array}\right]\left[\begin{array}{l}R \\ G \\ B\end{array}\right]\end{aligned}$

La Tabla 3 presenta los resultados de aplicar la matriz de transformación obtenida y los datos usados en la construcción del modelo.

Tabla 3. Transformación R, G, B - X, Y, Z

\begin{tabular}{cccccccccc}
\hline Fuente & $X_{R}$ & $Y_{R}$ & $Z_{R}$ & $R$ & $G$ & $B$ & $X_{E}$ & $Y_{E}$ & $Z_{E}$ \\
\hline$L E D_{1}$ & 101,2 & 100,0 & 32,0 & 255,0 & 241,2 & 77,8 & 105,4 & 106,8 & 27,5 \\
$L E D_{2}$ & 85,7 & 100,0 & 86,1 & 149,2 & 255,0 & 165,0 & 77,0 & 91,7 & 85,3 \\
$F L U_{1}$ & 105,8 & 100,0 & 4,0 & 255,0 & 173,1 & 46,1 & 100,3 & 95,4 & 8,8 \\
$F L U_{2}$ & 86,8 & 100,0 & 90,8 & 183,7 & 255,0 & 182,3 & 91,1 & 104,0 & 92,4 \\
$F L U_{3}$ & 102,6 & 100,0 & 5,9 & 255,0 & 174,0 & 42,4 & 100,0 & 94,9 & 6,7 \\
$H A L_{1}$ & 98,2 & 100,0 & 30,5 & 255,0 & 199,0 & 88,1 & 105,3 & 104,3 & 32,9 \\
$H A L_{2}$ & 99,2 & 100,0 & 29,9 & 255,0 & 194,8 & 87,1 & 105,1 & 103,7 & 32,3 \\
$H A L_{3}$ & 103,9 & 100,0 & 35,8 & 255,0 & 170,4 & 92,1 & 105,0 & 102,1 & 34,8 \\
$I N C_{1}$ & 108,8 & 100,0 & 21,9 & 255,0 & 146,6 & 64,3 & 101,5 & 95,6 & 18,8 \\
$I N C_{2}$ & 104,1 & 100,0 & 29,1 & 255,0 & 164,5 & 77,4 & 103,3 & 99,3 & 26,4 \\
\hline
\end{tabular}

Posteriormente usando los valores estimados de $X, Y, Z$ $\left(X_{E}, Y_{E}, Z_{E}\right)$ se obtiene por mínimos cuadrados el modelo que permite estimar el valor de CRI. En la Tabla $4 C R I_{R}$ es el valor obtenido a partir del SPD y el $C R I_{E}$ el estimado con el sensor RGB TCS3414CS. 
Tabla 4. Estimación CRI

\begin{tabular}{cccccccc}
\hline Fuente & $X_{E}$ & $Y_{E}$ & $Z_{E}$ & $C R I_{R}$ & $C R I_{E}$ & err $_{a}$ & err \\
\hline$L E D_{1}$ & 105,4 & 106,8 & 27,5 & 62,9 & 62,6 & 0,4 & $-0,6$ \\
$L E D_{2}$ & 77,0 & 91,7 & 85,3 & 72,6 & 68,1 & 4,5 & $-6,3$ \\
$F L U_{1}$ & 100,3 & 95,4 & 8,8 & 63,6 & 65,8 & $-2,1$ & 3,4 \\
$F L U_{2}$ & 91,1 & 104,0 & 92,4 & 86,1 & 90,2 & $-4,1$ & 4,8 \\
$F L U_{3}$ & 100,0 & 94,9 & 6,7 & 63,6 & 63,4 & 0,2 & $-0,3$ \\
$H A L_{1}$ & 105,3 & 104,3 & 32,9 & 79,6 & 81,1 & $-1,5$ & 1,9 \\
$H A L_{2}$ & 105,1 & 103,7 & 32,3 & 80,1 & 81,8 & $-1,7$ & 2,1 \\
$H A L_{3}$ & 105,0 & 102,1 & 34,8 & 91,7 & 92,0 & $-0,3$ & 0,4 \\
$I N C_{1}$ & 101,5 & 95,6 & 18,8 & 87,5 & 83,9 & 3,6 & $-4,1$ \\
$I N C_{2}$ & 103,3 & 99,3 & 26,4 & 87,5 & 85,7 & 1,9 & $-2,1$ \\
\hline
\end{tabular}

\section{CONCLUSIONES}

En este trabajo se presentó el modelo de cálculo del CRI a partir de la SPD, se caracterizaron varias fuentes usadas comúnmente en ambientes residenciales y se presentó un modelo para estimar el valor del CRI con un sensor RGB de bajo costo. Los resultados muestran que la estimación tiene un error relativo inferior al $6.3 \%$ y el modelo reduce considerablemente la complejidad necesaria para estimar este parámetro.

El cálculo y la estimación de estos parámetros se desarrollan para mejorar el diseño de sistemas de iluminación inteligentes residenciales buscando estabilizar además de la potencia de la luz en interiores, los valores de cromaticidad para tener la respuesta ideal y usar sistemas de iluminación que permitan tener estas variables de control. La reducción en los costos de medida y la construcción de modelos de estimación simples, permiten realizar implementaciones de sistemas inteligentes que aportan a la mejora en la calidad de la iluminación y en la reducción del consumo de energía usando sistemas híbridos. 


\section{AGRADECIMIENTOS}

Este trabajo está enmarcado en el proyecto "Diseño e implementación de un sistema de control inteligente de iluminación con compensación de luz natural para reducir el consumo eléctrico en edificaciones" con código P12204, del Grupo Automática y Electrónica COL0053581 adscrito al Instituto Tecnológico Metropolitano, Medellín-Colombia.

\section{REFERENCIAS}

Amensour, M., Sánchez-zapata, E., Abrini, J., \& Sendra, E. (2010). Estabilidad del color en salchichas de pollo tipo Frankfurt adicionadas con extracto acuoso de hoja de Myrtus communis Color stability in Frankfurter chicken sausages added with Myrtus communis leaf aqueous extract, 43(2000), 251-257.

Ashibe, M., Miki, M., \& Hiroyasu, T. (2008). Distributed optimization algorithm for lighting color control using chroma sensors. In 2008 IEEE International Conference on Systems, Man and Cybernetics (pp. 174-178). IEEE. doi:10.1109/ICSMC.2008.4811270

ASSIST. (2010). Guide to Light and Color in Retail Merchandising (Vol. 8, pp. 1-16). Retrieved from http://www.lrc.rpi.edu/programs/solidstate/assist/

Botero Valencia, J. S., \& Delgado Trejos, E. (2009). Segmentación para la identificación y planeación de trayectorias de Robots en $2 \mathrm{D}$ sobre secuencias de video. In STSIVA 2009. Pereira.

Bouma, P. J. (1948). Physical Aspects of Colour: An Introduction to the Scientific Study of Colour Stimuli and Colour Sensations. Philips Industries.

CIE. (2013). Selected Colorimetric Tables. Viena. Retrieved from http://www.cie.co.at/index.php/LEFTMENUE/index.php?i_ca_id=298

Elvidge, C. D., Keith, D. M., Tuttle, B. T., \& Baugh, K. E. (2010). Spectral Identification of Lighting Type and Character. Sensors (Basel, Switzerland), 10(4), 3961-3988. doi:10.3390/s100403961

Fernández-Vázquez, R., Stinco, C. C., Escudero-Gilete, M. L., Meléndezmartínez, A. J., Heredia, F. J., \& Vicario, I. M. (2010). Estudio preliminar sobre la utilidad del color para clasificar los zumos de naranja según su elaboración. Óptica pura y aplicada, (2009), 245-249.

Lei, Z., Xia, G., Ting, L., Xiaoling, G., Qiao Ming, L., \& Guangdi, S. (2007). Color rendering and luminous efficacy of trichromatic and tetrachro- 
matic LED-based white LEDs. Microelectronics Journal, 38(1), 1-6. doi:10.1016/j.mejo.2006.09.004

Logadóttir, Á., Fotios, S. a., Christoffersen, J., Hansen, S. S., Corell, D. D., \& Dam-Hansen, C. (2013). Investigating the use of an adjustment task to set preferred colour of ambient illumination. Color Research \& Application, 38(1), 46-57. doi:10.1002/col.20714

Lopera, F. (2004). Evolución y Cognición. Revista Neuropsicología, Neuropsiquiatría y Neurociencias, 6, 27-34.

McCamy, C. (1992). Correlated color temperature as an explicit function of chromaticity coordinates. Color Research \& Application, 142-144. Retrieved from http://onlinelibrary.wiley.com/doi/10.1002/col.5080170211/abstract

Motonaga, Y., Matsumoto, T., \& Motonaga, N. (2010). Color chart of European Pear "Le Lectier"based on the color image analysis. In SICE Annual Conference (pp. 2455-2461). Retrieved from http://ieeexplore.ieee.org/xpls/abs_all.jsp?arnumber $=5602400$

Pandharipande, A., \& Caicedo, D. (2011). Daylight integrated illumination control of LED systems based on enhanced presence sensing. Energy and Buildings, 43(4), 944-950. doi:10.1016/j.enbuild.2010.12.018

Perales, E., Chorro, E., García-domene, M. C., Fez, M. D. De, Óptica, D., Anatomía, F., ... Oftalmológica, F. (2010). Características colorimétricas de una pantalla con tecnología OLED Colorimetric characteristics of an OLED display, 43(2), 173-180.

Rea, M. S., \& Freyssinier-Nova, J. P. (2008). Color rendering: A tale of two metrics. Color Research \& Application, 33(3), 192-202. doi:10.1002/col.20399

Sandhu, J. S., Agogino, A. M., \& Agogino, A. K. (2004). Wireless sensor networks for commercial lighting control: decision making with multiagent systems. In AAAI workshop on sensor networks (pp. 131-140). Citeseer. $\quad$ Retrieved from http://citeseerx.ist.psu.edu/viewdoc/download?doi=10.1.1.64.4931\&am p;rep=rep1\&amp;type=pdf

Sharma, G. (2003). Digital Color Imaging: Handbook (p. 797). CRC Press. Retrieved from http://books.google.com/books?id=OxlBqY67rl0C\&pgis=1 